Article

\title{
The Effect of Variations of Flow from Tributary Channel on the Flow Behavior in a T-Shape Confluence
}

\author{
Aliasghar Azma ${ }^{1, *}$ and Yongxiang Zhang ${ }^{2}$ \\ 1 College of Architecture and Civil Engineering, Beijing University of Technology, Beijing 100124, China \\ 2 Institute of Water Resources and Engineering Municipal Engineering Department, College of Civil \\ Engineering and Architecture, Beijing University of Technology, Beijing 100124, China; yxzhang@bjut.edu.cn \\ * Correspondence: aa.azma1990@emails.bjut.edu.cn
}

Received: 22 April 2020; Accepted: 18 May 2020; Published: 21 May 2020

\begin{abstract}
Channel confluences are of the common structures in fluid transport channels. In this study, a series of numerical simulations were performed, utilizing a 3D code to investigate the reaction of the flow parameters and vortical structure to the variations in flow discharge and its Froude number from both main channel and tributary branch in a T-shape junction. The code was calibrated with the experimental data. Parameters, including the velocity, the turbulence energy, stream surface profile, head losses, and the transverse flow motions, were considered in different situations. It was concluded that increasing the ratio of discharge of flow from side-channel to the main channel ( $\left.\mathrm{Q}^{*}\right)$ increased the area and power of the recirculation zone, as well as the width of separation plate downstream of the confluence, while it reduced the area of the stagnation zone (or the wake vortex) within the side-channel. It was also indicated that increasing the discharge ratio from side-channel resulted in an increase in the upstream water level in the main channels, which was dependent on the upstream discharge.
\end{abstract}

Keywords: channel confluence; turbulent structure; the separation zone

\section{Introduction}

A confluence is considered to be a point where two various flow characteristics join together and make a single flow, which causes significant turbulence characteristics. Confluences and diversions are the most common occurrences along both natural rivers and artificial channels. Different parameters, such as geometrical properties (i.e., channel cross-section, the angle between the branch and the main channel), and hydraulic parameters (i.e., Froude number of the flows, the discharge ratio of the branch to the main channel), have been found to be effective on the flow behavior and its effect on the loose bed of earth channels. The interaction between the main channel and the side inflow creates a 3D flow pattern with separation zones at both side and main channels. It also causes a significant increase in flow speed along with a drop in flow surface, which leads to a large amount of scouring at the opposite side of junction point and sedimentation adjacent to the inner wall of the main channel [1]. Generally, a comprehensive understanding of the sediment and junction or the interaction and behavior of diversion structures requires a thorough knowledge of the three-dimensional flow behavior around these structures [2].

Numerous investigations have been carried out to study the flow pattern through the junction of the channel, and there is a plethora of experimental, analytical, and numerical research on different aspects of flow behavior in such structures. From the very beginning research performed on confluences, various researchers, such as Taylor [3], Webber and Greated [4], Shumate [5], Mignot et al. [6], 
have considered the ratio of side-channel to main channel discharge as a key parameter of the flow behavior in channel confluences and diversions. Webber et al. [7] studied the pattern of the flow in a $90^{\circ}$ open channel via conducting a series of laboratory experiments. They considered the flow behavior in different ratios of side-channel discharge to the total channel varying from 0.08 to 0.9 and discussed the effect of discharge ratios on flow patterns in the channel. They provided significant data on the velocity domain, turbulence stresses, and water surface elevations. Liu et al. [8] experimentally studied the flow patterns and sediment transport in a confluence. They stated that increasing the ratio of tributary flow to total flow discharge restricted the development of the separation zone. They also suggested that the ratio of bedload transport and the scouring intensity relied mainly on the discharge ratio from the tributary channel. Zhang et al. [9] used 3D k- $\omega$ turbulence model and the Reynolds averaged Navier-Stokes equations to investigate the effect of the ratio of the flow in upstream of the main channel to the overall flow downstream on the separation zone's shape and size, the contraction coefficient, and cross-sectional mean flow angle for a $90^{\circ}$ open-channel with the same width. They concluded that larger discharge ratios of side-channel to the main channel might result in less uniformly distribution of flow and velocity profiles. They also stated that despite the variations in length and width of the recirculation zone in the main channel, its shape index remained almost constant. By utilizing a hybrid Reynolds averaged Navier Stokes (RANS)-based large eddy simulation (LES) model, Zeng and Li [10] simulated flow in an open-channel T-junction. Their results were far accurate and precise compared to the RANS results, which clearly indicated the preference of this model. Ting et al. [11] used a 3D K- $\omega$ model to simulate the flow for the $90^{\circ}$ open-channel junction with the same width and investigated the influence of the ratio of the discharge on the separation zone, the cross-sectional mean velocity of the flow, and the coefficient of the contraction. Riley and Rhoads [12] investigated both flow pattern and morphology of the bed; furthermore, they studied the impacts of the curvature of the channel and the angle of the confluence on the confluence morpho-dynamics. Mohammadiun et al. [1] performed a series of numerical simulations and studied the effect of different geometrical modifications on the flow behavior in a 90-degree junction. Modifications included adding converging and diverging arcs at corners of the junction, as well as the elimination of recirculation zones by making arc shape modifications in the main channel based on the idea of streamlinization. Their results indicated that the modifications successfully improved the flow pattern and eliminated the formation of recirculation areas. Liu et al. [13] investigated the flow-sediment interaction in confluences with different junction angles. Their results showed that the sediment transport rate increased with increasing the inflow ratio and junction angle. Biswal et al. [14] studied the hydraulics of flow in a right-angled confluence in the case of compound channels. They stated that the flow in such channels was highly different from that in simple channels. Sharifipour et al. [15] used a k- $\omega$ turbulence model to simulate the effect of tributary channel width on the flow characteristics in the main channel. It was indicated that increasing the ratio between tributary channel widths to main channel width from 0.5 to 2 might lead to a decrease in the effect of tributary flow on the characteristics of the main channel flow. Schindfessel et al. [16] numerically investigated the confluence flow patterns with a predominant fraction $(90 \%<)$ of the total flow consisted of the tributary inflow. It was found that the tributary flow collided the opposing bank in the predominant tributary flow, triggering a recirculating eddy in the upstream channel of the confluence. Wang et al. [17] experimentally investigated the flow confluences on the natural rivers. They studied the flow junction impact on the stage-discharge relationship at the confluence. Sui and Huang [18] numerically simulated the separation zone. This quasi-2-D simulation was done in the high Reynolds number and using the LES model. The comparison between numerical results and the experimental measurements showed perfect acceptance. Ramos et al. [19] compared the accuracy of flat and rigid-lid techniques using the LES turbulence model for the simulation of free-surface flow in channel junctions. In another research, Ramos et al. [20] studied the effect of bed elevation discordance of the flow patterns and flow turbulent structure in a right-angled confluence.

Reviewing literature reveals that there is a significant amount of data on the different aspects of flow behavior in different geometrical and hydraulic conditions of junction's performance; yet, 
various researchers are working on different aspects of the flow and sediment transport within confluences. Several researchers have considered the effect of the ratio of tributary channel discharge to total channel discharge. They have stated this parameter as the governing parameter in sediment scour and flow structure at confluences. Nevertheless, different combinations of discharges from the tributary channel and main channel with a constant total Froude number-at downstream section after confluence- could be formed. The present study made efforts to explain the differences in flow patterns and their power induced by variations in discharges coming from the tributary channel and main channel in conditions with constant or varying total discharge. Sediment transport and sedimentation is a direct consequence of flow patterns and vortex structure above the bed and close to sensitive points. Hence, this paper made efforts to investigate the 3D behavior of flow at a T-shape junction in different ratios of side channels discharge to the main channel.

\section{Materials and Methods}

\subsection{Laboratory Model}

The present study utilized the experimental data of Webber et al. (2001) to calibrate and validate numerical results. The experimental model consisted of the main channel with $21.95 \mathrm{~m}$ length, $0.91 \mathrm{~m}$ width $(\mathrm{Wc})$, and $0.51 \mathrm{~m}$ height, as well as a side-channel with the same height and width and $3.66 \mathrm{~m}$ long. The total flow discharge of $Q_{t}=0.17 \mathrm{~m}^{3} / \mathrm{s}$ with $\mathrm{h}=0.296 \mathrm{~m}$, resulting in a Froude number of $\mathrm{Fr}_{\mathrm{t}}=0.37$, was used in the calibration test.

\subsection{Numerical Model}

This study utilized the FLOW-3D numerical model as a solver of the Navier-Stokes equation to calculate fully 3D flow patterns at the channel junction.

\subsection{Governing Equations}

The governing equations comprised the momentum and continuity equations. The continuity equation, regardless of the density of the fluid in the form of Cartesian coordinates $x, y$, and $z$, was as follows:

$$
V_{\mathrm{F}} \frac{\partial \rho}{\partial t}+\frac{\partial}{\partial x}\left(u A_{x}\right)+\frac{\partial}{\partial y}\left(v A_{y}\right)+\frac{\partial}{\partial z}\left(w A_{z}\right)=\frac{R_{\text {sor }}}{\rho}
$$

where $u, v$, and $w$ are the components of velocity in the $x, y$, and $z$ directions, respectively; $A_{x}, A_{y}$, and $A_{z}$ are the surface flow fractions in the $x, y$, and $z$, respectively; $V_{\mathrm{F}}$ is the flow volume fraction; $\rho$ is the fluid density; $t$ is time, and $R_{\text {sor }}$ is the mass source. Equation (2) is the momentum equation in three dimensions:

$$
\begin{aligned}
& \frac{\partial u}{\partial t}+\frac{1}{V_{\mathrm{F}}}\left(u A_{x} \frac{\partial u}{\partial x}+v A_{y} \frac{\partial u}{\partial y}+w A_{z} \frac{\partial u}{\partial z}\right)=-\frac{1}{\rho} \frac{\partial \rho}{\partial x}+G_{x}+f_{x} \\
& \frac{\partial v}{\sigma t}+\frac{1}{V_{\mathrm{F}}}\left(u A_{x} \frac{\partial v}{\partial x}+v A_{y} \frac{\partial v}{\partial y}+w A_{z} \frac{\partial v}{\partial z}\right)=-\frac{1}{\rho} \frac{\sigma \rho}{\partial y}+G_{y}+f_{y} \\
& \frac{\partial w}{\partial t}+\frac{1}{V_{\mathrm{F}}}\left(u A_{x} \frac{\partial w}{\partial x}+v A_{y} \frac{\partial w}{\partial y}+w A_{z} \frac{\partial w}{\partial z}\right)=-\frac{1}{\rho} \frac{\sigma \rho}{\partial z}+G_{z}+f_{z}
\end{aligned}
$$

where $G_{x}, G_{y}$, and $G_{z}$ are the accelerations due to the gravity in the $x, y$, and $z$ directions, respectively; $f_{x}, f_{y}$, and $f_{z}$ are the accelerations due to viscosity in the $x, y$, and $z$ directions, respectively.

\subsection{Turbulence Modeling}

The utilized turbulence models in this study were the $k-e, k-w$, and $k-\varepsilon$ renormalized group (RNG) models. The $k-\varepsilon$ model is highly sophisticated, widely-used and consisted of two transport equations: 
one for turbulent kinetic energy $k$, and the other for its dissipation $\varepsilon$ [20]. The 3D governing equations could be expressed as follows [21,22]:

$$
\begin{gathered}
\frac{\partial}{\partial t}(\rho k)+\frac{\partial}{\partial x}(\rho k u)=\frac{\partial}{\partial x}\left[\left(\mu+\frac{\mu_{t}}{\sigma_{k}}\right) \frac{\partial k}{\partial x}\right]+G_{k}+G_{b}-\rho \varepsilon-Y_{M} \\
\frac{\partial}{\partial t}(\rho \varepsilon)+\frac{\partial}{\partial x}(\rho \varepsilon u)=\frac{\partial}{\partial x}\left[\left(\mu+\frac{\mu_{t}}{\sigma_{\varepsilon}}\right) \frac{\partial \varepsilon}{\partial x}\right]+C_{1 \varepsilon} \frac{\varepsilon}{k}\left(G_{k}+C_{3 \varepsilon} G_{b}\right)-C_{2 \varepsilon} \rho \frac{\varepsilon^{2}}{k} \\
\mu_{t}=\rho C_{\mu} \frac{k^{2}}{\varepsilon}
\end{gathered}
$$

where $\mu_{t}$ represents eddy viscosity, $\mathrm{G}_{\mathrm{b}}$ and $\mathrm{G}_{\mathrm{k}}$ are generations of the turbulent kinetic energy due to buoyancy and mean velocity gradients, respectively; $\mathrm{Y}_{\mathrm{M}}$ is the contribution of the fluctuating dilatation incompressible turbulence to the overall dissipation rate; $\mathrm{C}_{1 \varepsilon}, \mathrm{C}_{2 \varepsilon}, \mathrm{C}_{3 \varepsilon}$, and $C_{\mu}$ are constants (1.44, 1.92, 0.09, and 0.09 respectively); $\sigma_{\mathrm{k}}$ and $\sigma_{\varepsilon}$ are turbulent Prandtl numbers for $k$ and $\varepsilon$ (1.0 and 1.3, respectively). The RNG model used equations analogous to the equations for the $k-\varepsilon$ model. However, constants that were found empirically in the standard $k-\varepsilon$ model were derived explicitly in the RNG model. Generally, the RNG model has wider applicability than the standard $k-\varepsilon$ model. In particular, the RNG model is known to present more accurately for low-intensity turbulence flows and flows with strong shear regions [2].

\subsection{Boundary Conditions and Gridding}

In this study, a structured non-uniform block of mesh was used to simulate the main and side channels. The meshes were more intense in the vicinity of the confluence to increase the accuracy of computations. Boundary conditions included inflow discharge for the main channel and the lateral channel entrance, constant level outflow for the main channel outlet, wall for bed and the right boundaries of the main channel, and symmetry for the top border. Other required wall conditions were produced using solid components. Given that using a single mesh domain caused two large empty domains at the sides of the lateral channel, these parts were eliminated from calculations using void or space components. Considering restrictions in the available processing power, the main mesh block was non-uniformly identified in order to increase the accuracy at the vicinity of the junction and reducing the number of mesh cells in less important areas. Considering the limitations in processing power (Core i7 CPU-turbo speed of up to $4.0 \mathrm{GHz}$ ), a trial and error process was performed to find a proper balance between the consumed simulation time and the resulted accuracy of the simulations. Different mesh sizes were tested; however, a severe increase in computational time-due to decreased mesh sizes-as well as the increased number of cells, restricted the use of meshes with side dimensions less than $4 \mathrm{~mm}$. Thus, a mesh domain, with $17 \mathrm{~m}$ length, $0.6 \mathrm{~m}$ width, and $0.4 \mathrm{~m}$ height and consisting of 2,524,480 cells, was opted to simulate the channels. The mesh domain was composed of 320 cells in the x-direction, 161 in the y-direction, and 49 cells in the z-direction. Neglecting the void spaces, all active cells were 1,839,166. As mentioned above, the cell sizes were reduced near the walls and bed to $4 \mathrm{~mm}$. Besides, the same cell size was identified at the corners of the junction and the space of flow mixing in order to increase the accuracy of the calculations. The plan and side view of the mesh domain, as well as the boundary conditions, are plotted in Figure 1. 


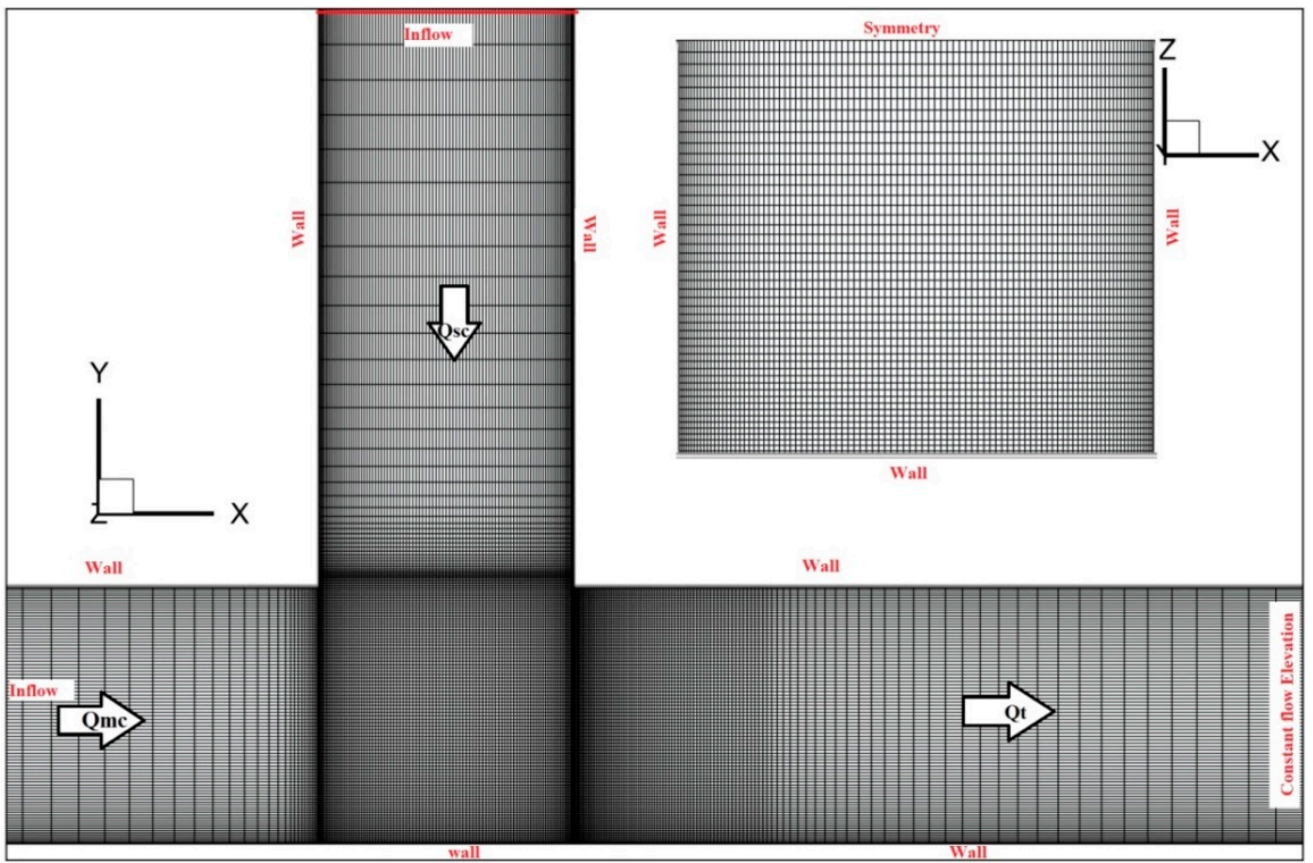

Figure 1. Plan and front view of mesh domain along with boundary conditions.

\section{Model Verification}

A first-order expansion of the momentum advection, the implicit generalized minimal residual method (GMRES) with an allowed number of 120 internal iterations in each step, an initial time step of 1E-6, and the fractional area-volume obstacle representation (FAVOR) method for estimation of the free surface were used to estimate the momentum and pressure field. The verification of the model was performed using experimental data from Webber et al. (2001). The simulations were performed with three turbulence models of $k-\omega, \mathrm{K}-\epsilon$ standard, and k- $\epsilon$ (RNG). The accuracy of the results of the models in terms of statistical error assessment criteria in the estimation of the $\mathrm{U}$ component of velocity was compared. Consequently, the K- $\epsilon$ standard model (with $\mathrm{R}^{2}=0.93, \mathrm{MAE}=0.13$, and RMSE $=0.26$ ) with less consumed time of simulation compared to RNG (with $\mathrm{R}^{2}=0.91$, MAE 0.15, and RMSE $=0.29$ ) and $\mathrm{k}-\omega$ (with $\mathrm{R}^{2}=0.93, \mathrm{MAE}=0.15$, and RMSE $=0.25$ ) was opted as the optimum turbulence model. A comparison of the stream-wise component of velocity $(\mathrm{U})$ calculated with the computational fluid dynamics (CFD) code with that of experimental data showed acceptable accuracy of the numerical results (see Figures 2 and 3).
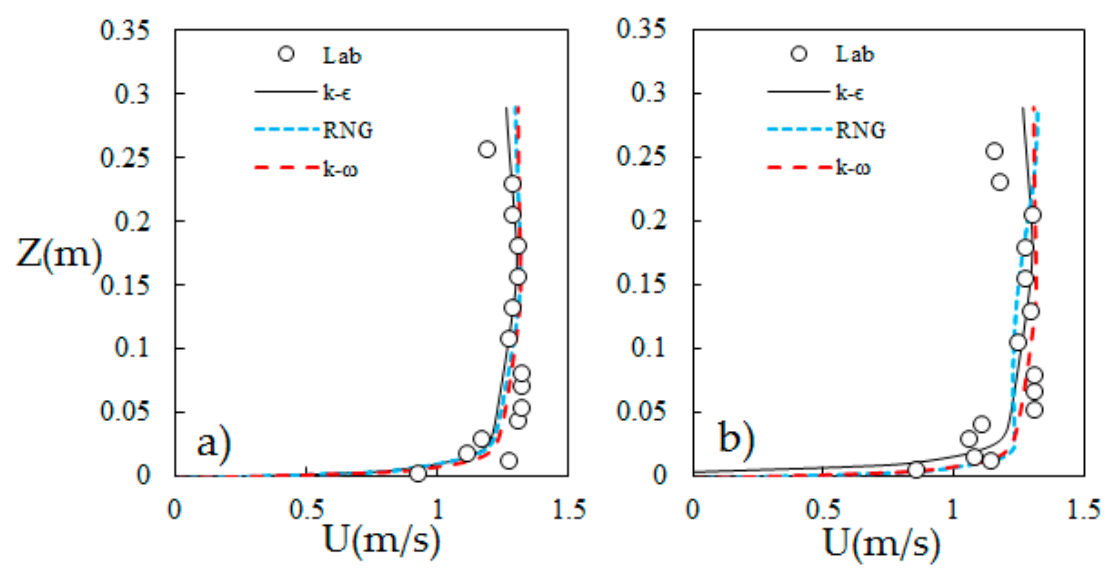

Figure 2. A comparison of the accuracy of velocity calculations from different turbulence models in estimating U-component of velocity at $\mathrm{x}=8.82\left(\mathrm{x}^{*}=2 \mathrm{Wc}\right)$ and $(\mathbf{a}) \mathrm{y}=0.25 \mathrm{Wc}$, and (b) $\mathrm{y}=0.5 \mathrm{Wc}$. 

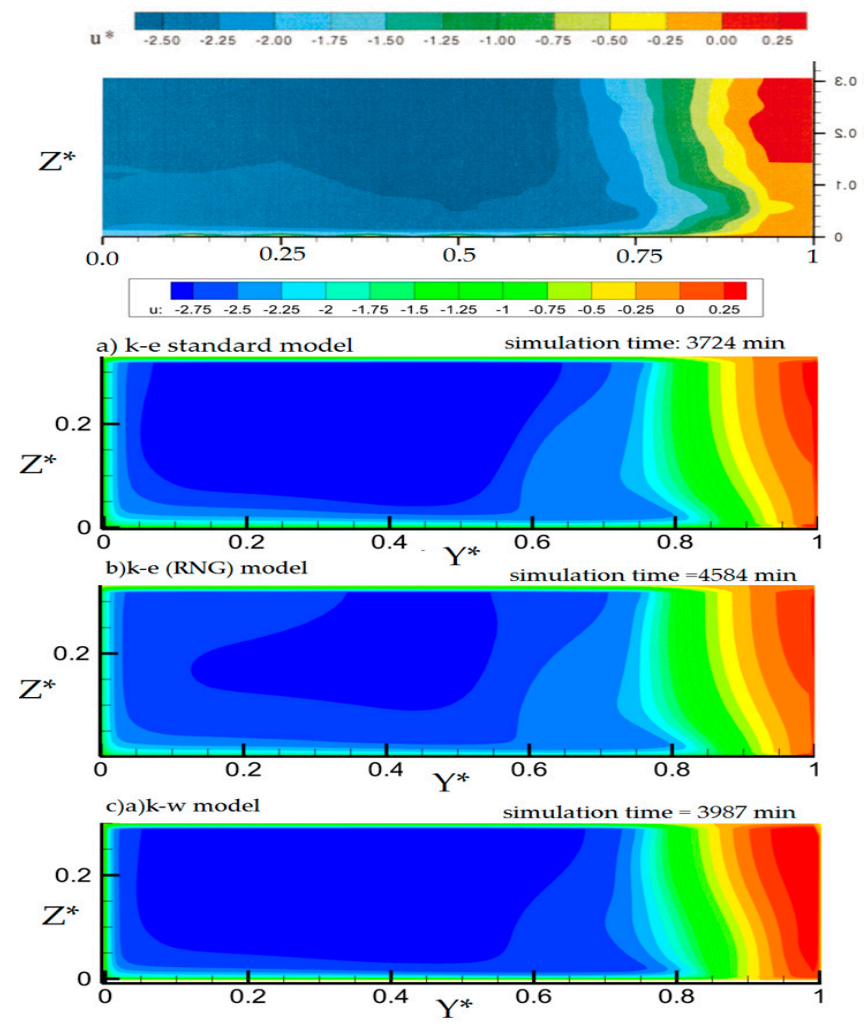

Figure 3. The validation of transverse velocity distribution at $x=8.82\left(x^{*}=2 \mathrm{Wc}\right)$.

\section{Simulation Scenarios}

A total number of seven different conditions were simulated with different ratios of side-channel to main channel discharge. Table 1 presents the simulation conditions and discharge ratios. Various researchers have concluded that the ratio between discharges is a key parameter. Still, some studies have also stated that the cross-sectional area of channels is a dominant parameter, affecting the flow behavior. On the other hand, changing the width of either of channels with the same geometry will have a significant effect on the flow structure. Hence, considering the ratio between Froude number of flow in side-channel $\left(\mathrm{Fr}_{\mathrm{sc}}\right)$ to Froude number of flow in the main channel $\left(\mathrm{Fr}_{\mathrm{mc}}\right)$, as well as the Froude number of the overall flow after the confluence $\left(\mathrm{FR}^{*}\right)$, as varying parameters in simulation scenarios would provide better understanding and could result in better and more generalized conclusions.

Table 1. Simulation cases.

\begin{tabular}{ccccccccc}
\hline Case No. & $\mathbf{Q}_{\mathbf{s c}}(\mathbf{L i t} / \mathbf{s})$ & $\mathbf{Q}_{\mathbf{m c}}(\mathbf{L i t} / \mathbf{s})$ & $\left.\mathbf{Q}_{\mathbf{t}} \mathbf{( L i t} / \mathbf{s}\right)$ & $\mathbf{Q}^{*}=\mathbf{Q}_{\mathbf{s c}} / \mathbf{Q t}$ & $\mathbf{F r}_{\mathbf{s c}}$ & $\mathbf{F r}_{\mathbf{m c}}$ & $\mathbf{F r}_{\mathbf{t}}$ & $\mathbf{F r}^{*}=\mathbf{F r}_{\mathbf{s c}} / \mathbf{F r}_{\mathbf{m c}}$ \\
\hline 1 & 35 & 127 & 162 & 0.22 & 0.07 & 0.26 & 0.33 & 0.28 \\
2 & 42 & 127 & 169 & 0.25 & 0.09 & 0.26 & 0.34 & 0.33 \\
3 & 49 & 127 & 176 & 0.28 & 0.10 & 0.26 & 0.36 & 0.39 \\
4 & 56 & 127 & 183 & 0.31 & 0.11 & 0.26 & 0.37 & 0.44 \\
5 & 56 & 115 & 171 & 0.33 & 0.11 & 0.23 & 0.35 & 0.49 \\
6 & 56 & 106 & 162 & 0.35 & 0.11 & 0.22 & 0.33 & 0.53 \\
7 & 56 & 95 & 151 & 0.37 & 0.11 & 0.19 & 0.31 & 0.59 \\
\hline
\end{tabular}

$\mathrm{Q}_{\mathrm{sc}}$ is the discharge from the side-channel. $\mathrm{Q}_{\mathrm{mc}}$ is the discharge from the upstream main channel. $\mathrm{Q}_{\mathrm{t}}$ is the total discharge after confluence. $\mathrm{Q}^{*}$ is ratio between discharge of tributary flow and the main channel flow. $\mathrm{FR}^{*}$ is the ratio between Froude number of tributary flow and the main channel flow. 


\section{Results and Discussion}

\subsection{Velocity Distribution}

The distribution of the stream-wise component of velocity (U) and the normal component of velocity (V) at $\mathrm{x}-\mathrm{y}$ plane around the confluence structure and along the main channel are presented in Figures 4 and 5, respectively, for three different cases, including case (1) with $\mathrm{Fr}^{*}=0.28$, case (4) with $\mathrm{Fr}^{*}=44$, and case (7) with $\mathrm{Fr}^{*}=0.59$. The results were presented in four different depths, including $\mathrm{z}=0.01 \mathrm{~m}$ (near the bed), $\mathrm{z}=0.08 \mathrm{~m}, \mathrm{z}=0.17 \mathrm{~m}$, and $\mathrm{z}=0.27$, close to the water surface. The other cases were not shown here due to the similarity between results. As expected, there was a significant difference in maximum velocity between case 1 (Figure $4 \mathrm{a}-\mathrm{d}$ ) and case 2 (Figure $4 \mathrm{e}-\mathrm{h}$ ). This difference was a direct consequence of increased discharge from side-channel in case 4 compared to case 1 , as well as the reduced width of the main channel after confluence due to the increase in the size of the recirculation zone, and agreed with the increased Froude number of total discharge. Unexpectedly, comparing case 1 to case 7 indicated that the velocity of flow after the confluence in case 7 was higher than that in case 1, especially near the bed and close to the surface, although the overall discharge in case (7) was less than that in case 1 (up to 7\%). Besides, the high-velocity zone moved downstream. Yet, it could be seen that the velocity gradient toward the recirculation zone at the downstream corner of confluence was more intense in case (1) compared to the case (7), which was caused by increased discharge and total Froude number of the tributary inflow.

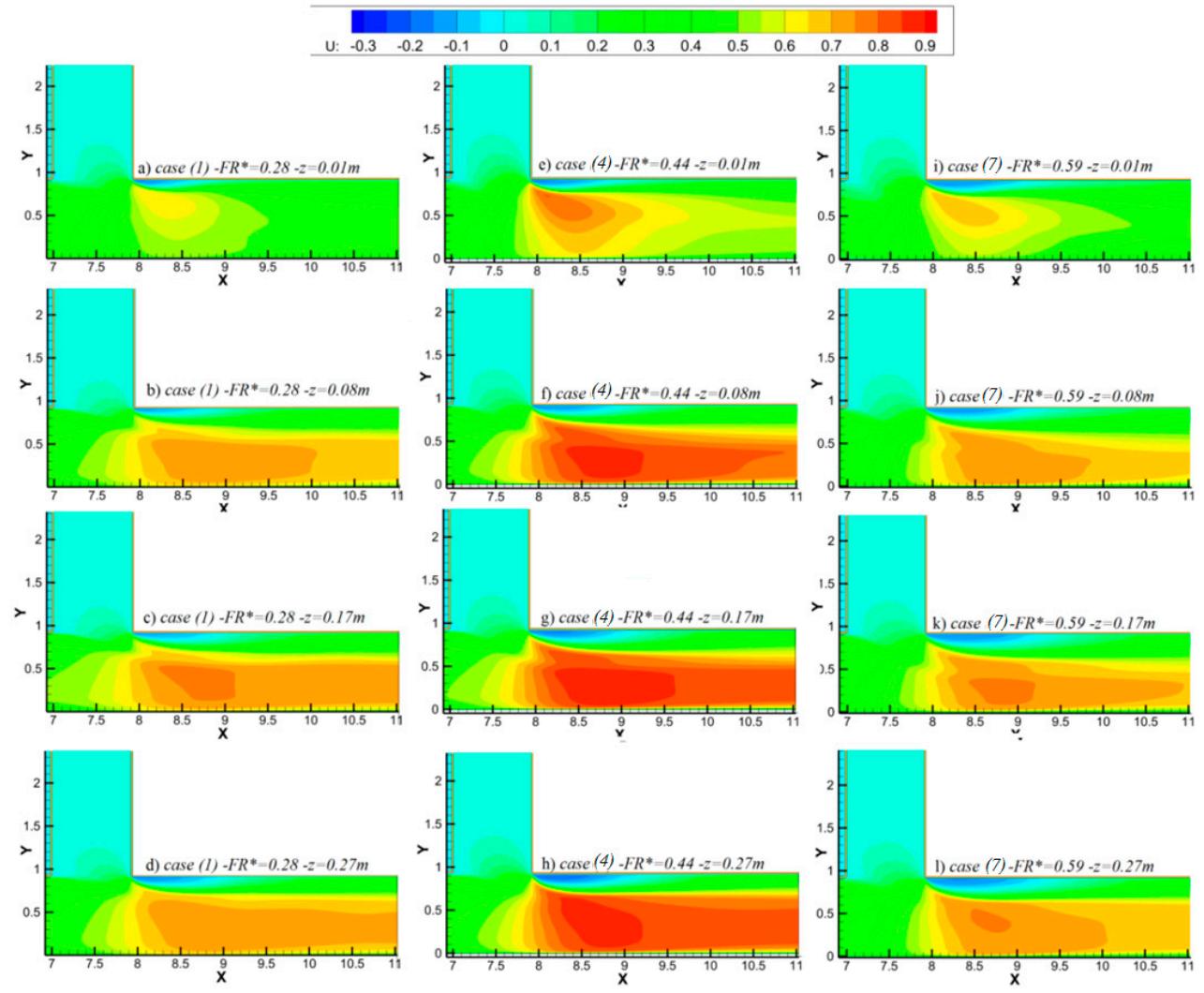

Figure 4. The distribution of the stream-wise component of velocity (U) for (a-d) case 1, and (e-h) case 4, and $(\mathbf{i}-\mathbf{l})$ case 7 . 

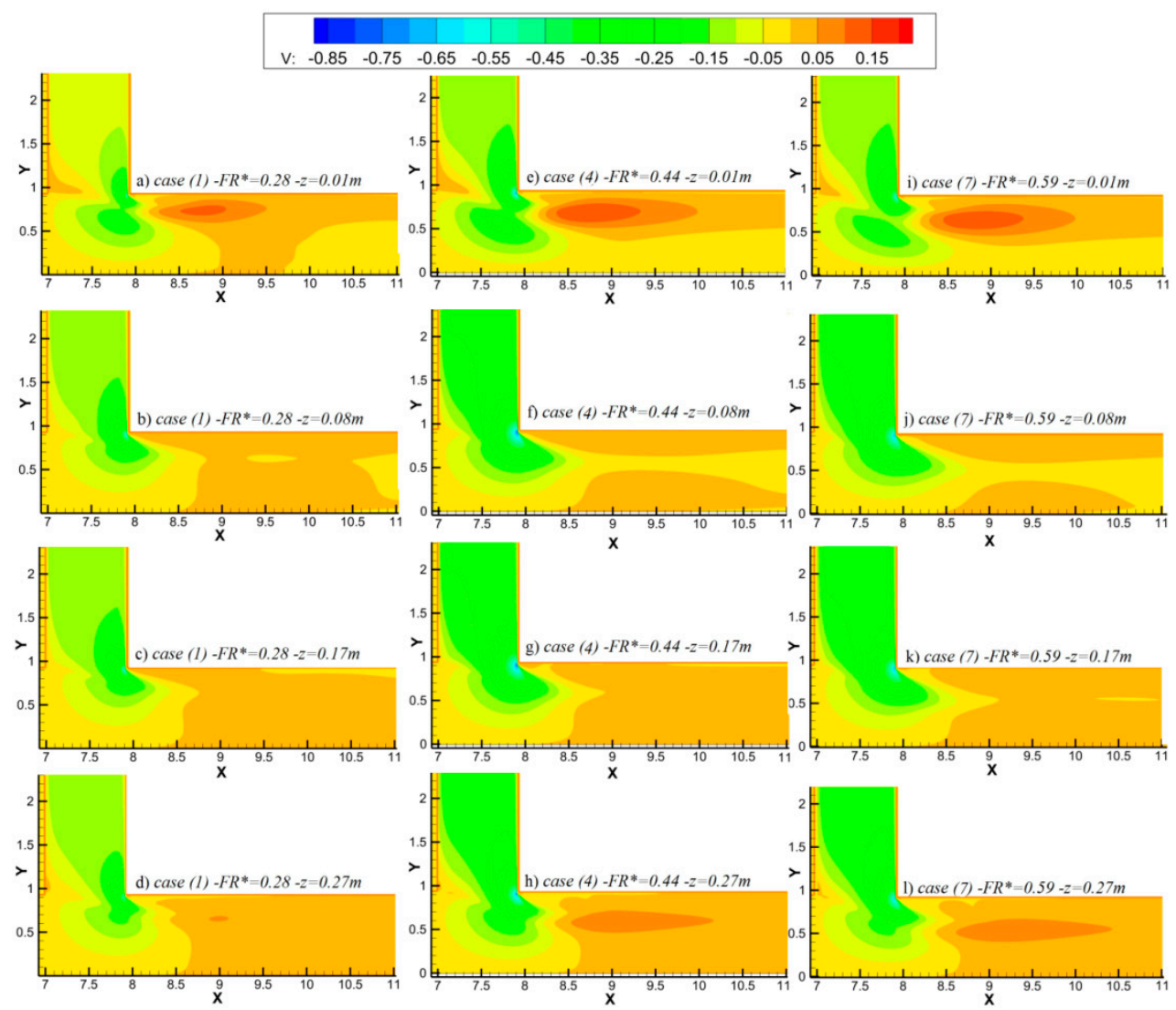

Figure 5. The distribution of the normal component of velocity (V) for (a-d) case 1, and (e-h) case 4, and (i-1) case 7 .

The same conclusion could be inferred from the distribution of the normal component of velocity in Figure 5. The V velocity gradient in case 7 was more intense than that in case (1) despite the reduced discharge and total Froude number of the main channel after confluence $\left(\mathrm{Fr}_{t}=0.31\right.$ for case 7$)$ in comparison with other cases. It could be interpreted through the increased width of the recirculation zone in the channel in case (7) compared to other cases. Generally, it could be inferred that increasing the Froude number of the side-channel or the ratio between the Froude numbers of side-channel to the Froude number of the main channel might result in higher velocity gradients near the bed, which might result in increased sediment scour, as well as increased sedimentation due to the increased area of recirculation zone.

Figure 6 presents the effect of variations in discharges from the side and main channel on the stream-wise and normal component of velocity for all test cases in the main channel after confluence at $x=7.91 \mathrm{~m}$ at the downstream edge of the junction and at $x=8.35 \mathrm{~m}\left(0.5^{*}\right.$ Wc downstream of the junction) close to the flow surface. Generally, increasing the total discharge in cases 1 to 4 resulted in an increased stream-wise velocity $(U)$ throughout the channel cross-section at the junction point. But, decreased discharge of the main channel in cases 5 to 7 resulted in a reduced velocity profile. Yet, similarities between cases 1 to 6 and cases 2 to 5 could be seen due to the same total discharge after confluence (Figure 6a). It could be inferred that various combinations of side channel discharge $\left(Q_{\mathrm{sc}}\right)$ and that of main channel $\left(\mathrm{Q}_{\mathrm{mc}}\right)$ could result in relatively similar stream-wise velocity distribution (Figure 6a,c), yet, the main difference, which caused variations in flow patterns, was caused by significant fluctuations in $\mathrm{V}$. 

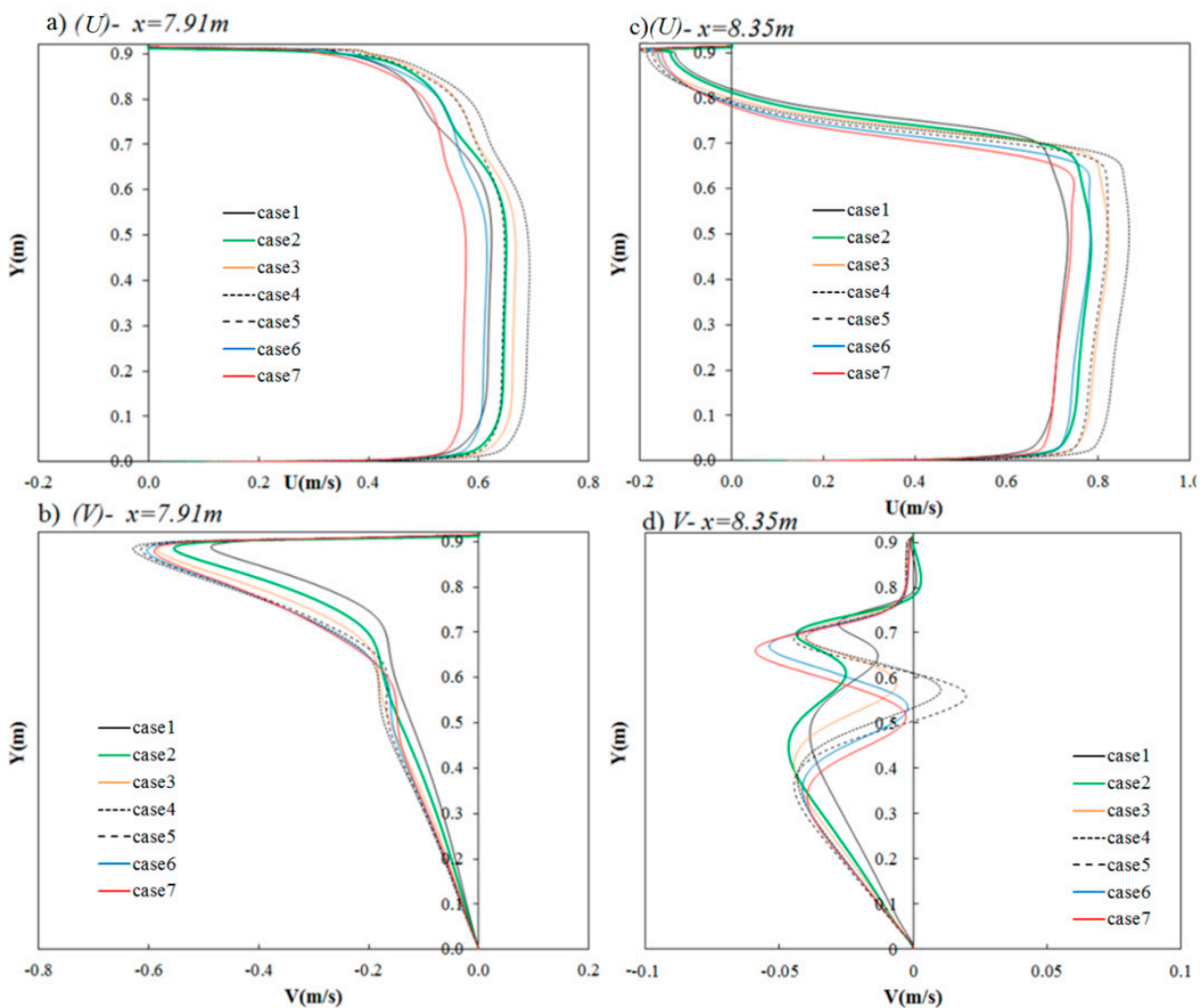

Figure 6. The comparison of velocity components at transverse planes (a) $U$ at $x=7.91,(\mathbf{b}) \mathrm{V}$ at $\mathrm{x}=7.91$, (c) $\mathrm{U}$ at $\mathrm{x}=8.35 \mathrm{~m}$, and (d) $\mathrm{V}$ at $\mathrm{x}=8.35 \mathrm{~m}$.

Considering the normal component of velocity $(\mathrm{V})$ at the junction section (Figure $6 \mathrm{~b}$ ), it was seen that for cases 1 to 4 , increasing the discharge from side-channel resulted in an increased $\mathrm{V}$ at the confluence. Besides, it was evident that the $\mathrm{V}$ velocity gradient decreased as the flow moved toward the opposite wall. Figure $6 \mathrm{~d}$ indicates the cross-sectional variations of the normal component of velocity (V) at $0.5 \mathrm{Wc}$ downstream of the confluence. Velocity gradients were significantly reduced compared to the junction section, yet there were distinct variations in different cases. Even though that the differences had no clear trend, yet, it could be inferred that by changing conditions from case 1 to case 7 (increasing the $\mathrm{Fr}^{*}$ ), the absolute value of $\mathrm{V}$ velocity close to the inner wall increased, which was equal to the increased width and length of recirculation zone.

\subsection{Flow Patterns and Vortical Structure}

\subsubsection{Flow Surface Profile}

Comparisons of the flow surface profiles for different cases at two transverse stations are presented in Figure 7. Generally, it was indicated that increasing $\mathrm{Fr}^{*}$ might increase the upstream water level before the confluence section. It should be noted that the upstream water level for cases 6 and 7 was higher than that for case 1 (Figure 7a). A surface drop at the tail of the confluence section was observed, which indicated sharper declines for cases with higher total discharges. Comparing similar cases, 1 with 6 and 2 with 5 (cases with same total discharge), indicated that the upstream level for cases with higher $\mathrm{Fr}^{*}$ (cases 5 and 6) was higher than that for cases with less $\mathrm{Fr}^{*}(1$ and 2 ) despite the similar discharges. Hence, it could be concluded that the upstream water level could be higher in cases with greater $\mathrm{Fr}^{*}$ but same or less total discharge, which indicated the governing role of discharge ratio in flow behavior, which surpassed the discharge values. 

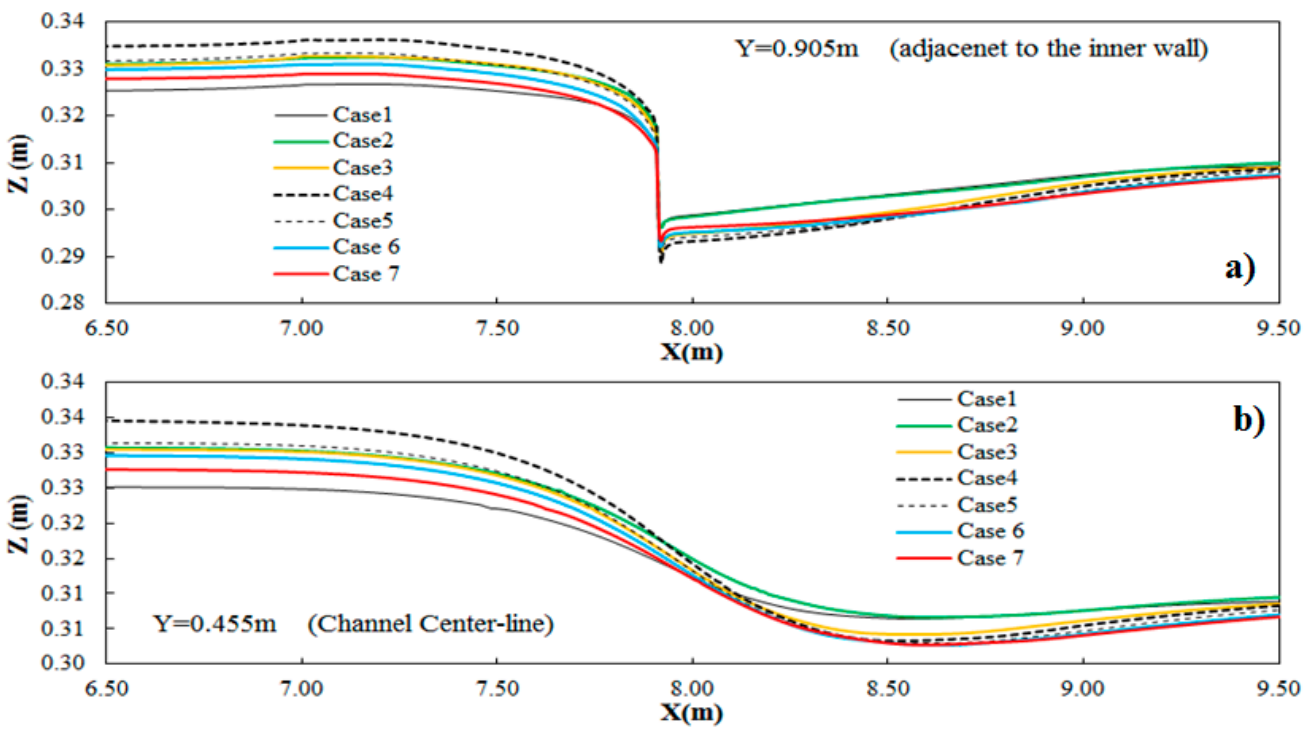

Figure 7. Longitudinal flow surface profiles at (a) $\mathrm{Y}=0.905 \mathrm{~m}$ and $(\mathbf{b}) \mathrm{Y}=0.455 \mathrm{~m}$ (channel centerline).

The difference in the downstream water level after the confluence was more significant. Surface drop in cases with less $\mathrm{Fr}^{*}$ was smaller and milder in profile, which suggested higher losses in cases with increased Fr*.

\subsubsection{Streamlines and Flow Patterns}

Generally, three significant vertical structures formed at a confluence, including a recirculation zone at the downstream corner of the junction adjacent to the inner wall of the main channel, a stagnation zone (or a wake vortex) at the inner corner of the tributary channel (Figure 8a,b), and the spiral movement and mixing of the tributary flow with the main channel flow (Figure 8c).

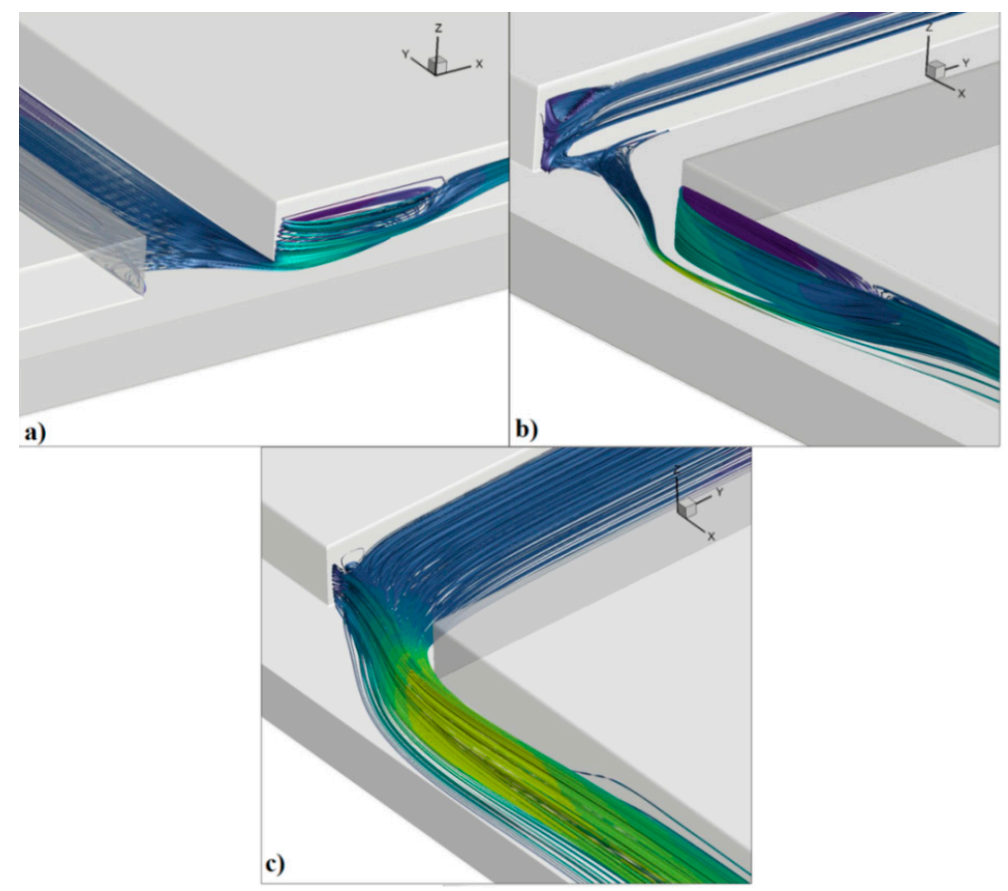

Figure 8. Three-dimensional streamlines at the confluence (a) downstream view of recirculation, (b) upstream view, and (c) tributary flow. 
Figure 9 presents the streamlines for cases 1,4 , and 7 in the mentioned depths in the previous section. It was clear that the size of the recirculation zone at the main channel increased as the Froude ratio increased from case 1 to case 7 , which was responsible for increased maximum velocity in case 7 compared to case 1 despite less discharge. The results were consistent with the findings of other researchers, such as [23] and [7]. With increasing the Froude ratio, loss of flow energy at the junction increased, as the momentum of tributary flow, which needed to be turned into the main channel flow, was increased. Consequently, higher energy drop might lead to a more significant separation zone [9]. Besides, an important dividing line starting from the upstream edge of the tributary channel was visible (where streamlines gather), which was located at different stations in different elevations of flow, indicating the section of shear flow at the mixing interface of tributary flow with the main stream.
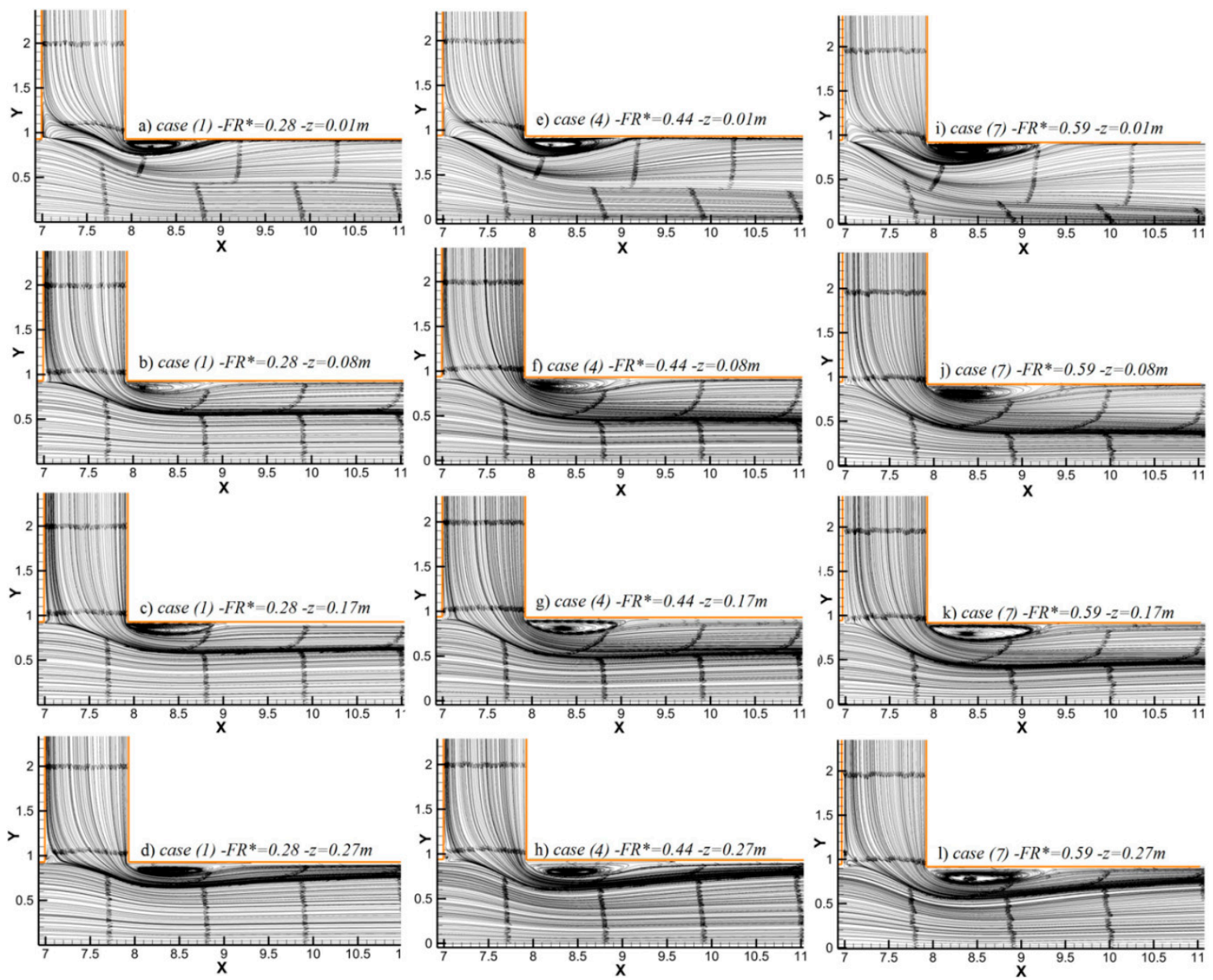

Figure 9. The effect of $\mathrm{Fr}^{*}$ on the flow pattern at different elevations for (a-d) case 1 and (e-h) case 4, and $(\mathbf{i}-\mathbf{l})$ case 7.

A significant stagnation zone at the upstream corner of the confluence could also be observed in lower discharge ratios, which was vanished as the discharge from side-channel increased. However, it could be noted that the stagnation zone in lower discharge ratios was, in fact, a wake vortex and is plotted in Figures $8 \mathrm{~b}$ and $9 \mathrm{c}, \mathrm{d}$.

As stated, the width and length of the recirculation zone increased with increasing the Froude ratio. Figure 10 represents the cross-sectional variations of the recirculation zone in each case from the bed to the flow surface. It was indicated that the cross-sectional area of the recirculation zone increased from bed to water surface in all cases. Besides, it was shown that the overall and the width of the recirculation zone at all elevations increased with increasing the Froude ratio from case 1 to case 7. 


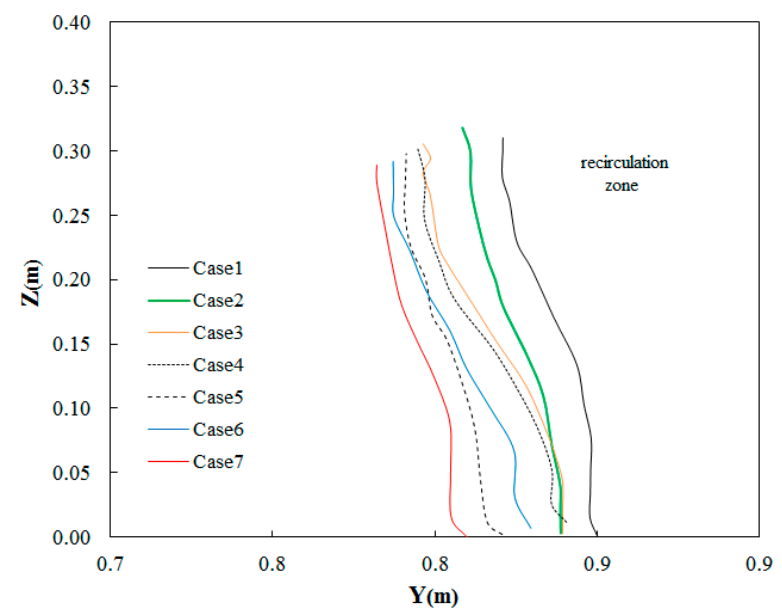

Figure 10. Transverse profile of recirculation zone at $x=8.35(x=0.5$ Wc downstream of confluence).

Figure 11 presents the length and width of the recirculation zone, as well as the shape index for each case and different $\mathrm{Fr}^{*}$ values. It was indicated that the length and width of the recirculation area increased with different slopes, resulting in a slightly increased shape index with increasing the $\mathrm{Fr}^{*}$, which could be assumed horizontal.

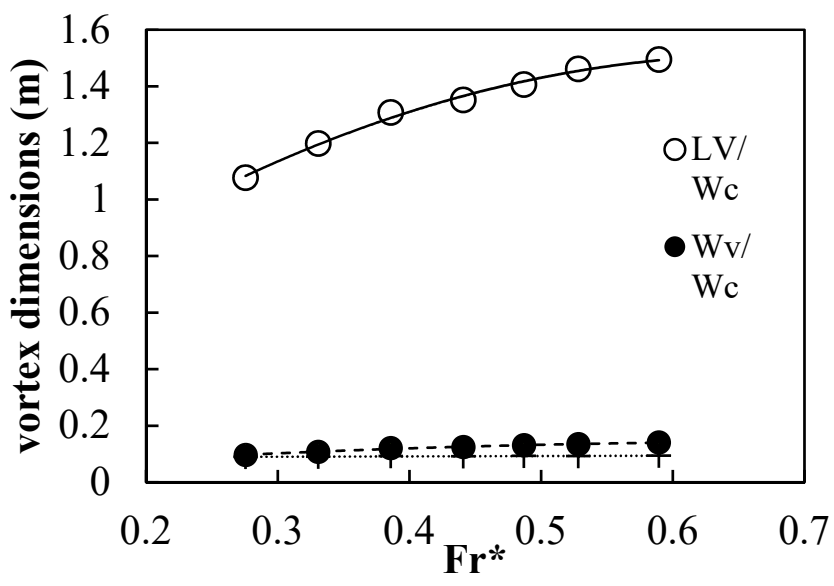

Figure 11. Transverse profile of recirculation zone at $x=8.35(x=0.5 \mathrm{Wc}$ downstream of confluence).

\subsection{Turbulent Kinetic Energy}

The distribution of the turbulent kinetic energy (TKE) at the transverse sections of $x=7.91$ and $x=8.35$ for all simulation cases are presented in Figure 12. It was indicated that a high positive gradient of TKE was formed at the downstream corner of the tributary channel. It could be seen that the maximum value of TKE was related to the case 4 with the maximum amount of total discharge and Froude number; for other cases, with decreasing the total flow discharge, the maximum values of TKE were reduced. It could be noted that the minimum value of TKE among all cases was related to case 1 and case 2 . A comparison between case 1 and 2 to case 6 and 7 showed that the maximum value of TKE in cases 6 and 7 with less total discharge and higher $\mathrm{Fr}^{*}$ was higher than that for cases 1 and 2, suggesting larger recirculation zones and higher ratios of turbulence despite less discharge (see Figure 12a). Considering the turbulent energy at $x=0.5 \mathrm{Wc}$ downstream of the junction, a relatively similar trend with that of the previous transverse section could be observed. 

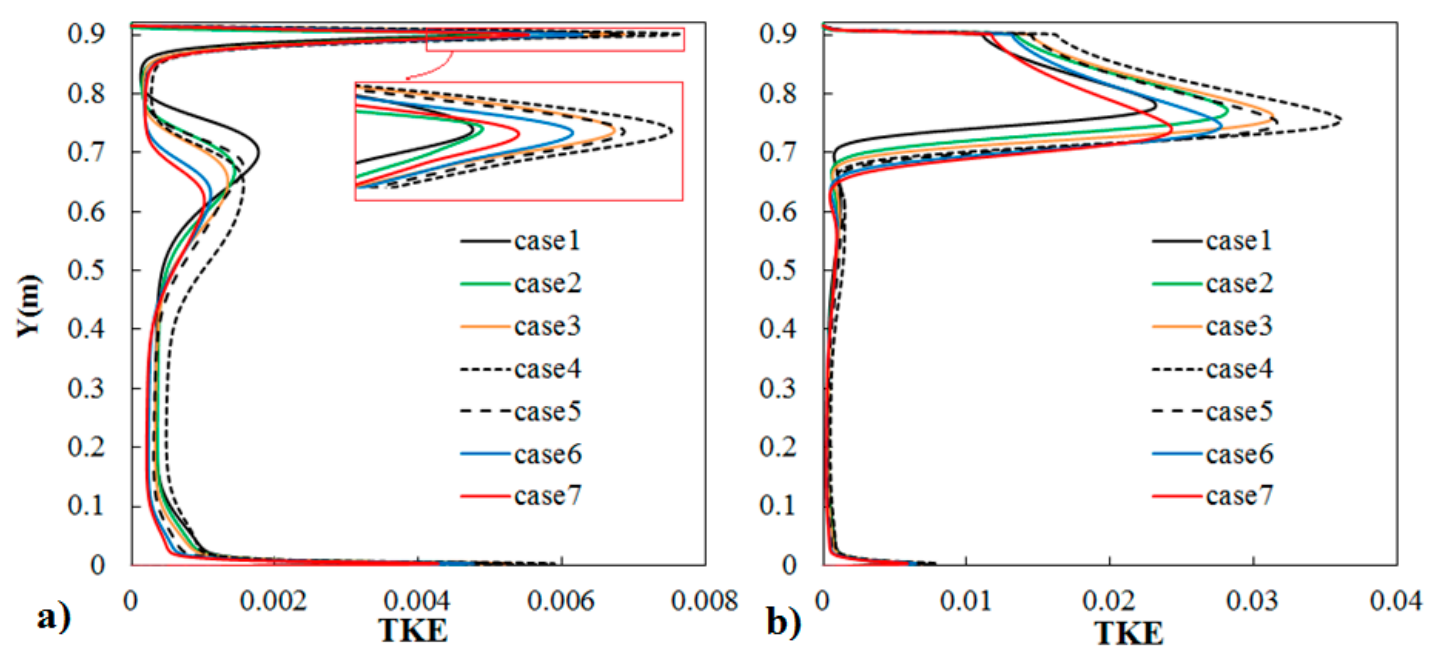

Figure 12. Transverse distribution of turbulent kinetic energy (TKE) at $(\mathbf{a}) \mathrm{x}=\mathrm{Wc}$ and $(\mathbf{b}) \mathrm{x}=1.5 \mathrm{Wc}$.

\section{Conclusions}

The present study investigated the effect of various combinations of flow from the tributary channel to the discharge from the main channel at a confluence. Seven different cases were simulated using a 3D CFD code, and the results were validated using the experimental data from the literature. Results indicated that the ratio between the Froude numbers of flows from the tributary channel to the flow of the main channel had a significant effect on the flow patterns and momentum exchange at the confluence.

Results indicated that the velocity gradient toward the recirculation was more intense in cases with a higher ratio of flow from the side-channel to the flow from the main channel, even in cases with less total discharge. It was due to the increased discharge and total Froude number of the tributary inflow. Besides, increasing the discharge ratio from side-channel resulted in an increase in the upstream water level in the main channel so that the upstream level for a flow with less total discharge but higher discharge ratio from tributary channel could be higher than that in cases with higher discharges but less portion coming from the tributary.

It could also be concluded that increasing the discharge from the tributary channel might result in higher velocity gradients near the bed, which, in turn, increased scour as well as sedimentation due to the expanded area of the recirculation zone. It also increased the dimensions of the recirculation zone as well as its power.

Finally, increasing the total discharge increased the turbulent energy within the domain, while for cases with constant total discharge, the values of TKE were higher for cases with less discharge from the main channel and more discharge coming from the tributary.

Author Contributions: Investigation, supervision, project administration, funding, Writing—Review \& Editing: Y.Z.; Methodology, Software, Writing-Original Draft Preparation, Formal Analysis, Data Curation: A.A.; All authors have read and agreed to the published version of the manuscript.

Funding: This research was funded by National Key Research and Development Program of China under No.2016YFC0401404.

Conflicts of Interest: The authors declare no conflict of interest.

\section{References}

1. Mohammaduin, S.; Salehi Neyshaburi, S.; Naser, G.; Parhizkar, H.; Vahabi, H. Effects of open-channel geometry on flow pattern in a $90^{\circ}$ junction. Iran. J. Sci. Tech. Trans. Civil Eng. 2015, 39, 559-573.

2. Karami, H.; Farzin, S.; Tavakol Sadrabadi, M.; Moazeni, H. Simulation of flow pattern at rectangular lateral intake with different dike and submerged vane scenarios. Water Sci. Eng. 2017, 10, 246-255. [CrossRef]

3. Taylor, E.H. Flow characteristics at rectangular open-channel junctions. Trans. ASCE 1944, 109, $893-902$. 
4. Webber, N.B.; Greated, C.A. An investigation of flow behaviour at the junction of rectangular channels. ICE Proc. 1966, 34, 321-334. [CrossRef]

5. Shumate, E.D. Experimental Description of Flow at an Open-Channel Junction. Master's Thesis, University of Iowa, Iowa City, IA, USA, 1998.

6. Mignot, E.; Vinkovic, I.; Doppler, D.; Riviere, N. Mixing layer in open-channel junctions. J. Environ. Fluid Mech. 2013, 14, 1027-1041. [CrossRef]

7. Weber, L.J.; Schumate, E.D.; Mawer, N. Experiments on flow at a $90^{\circ}$ open-channel junction. J. Hydraul. Eng. 2001, 127, 340-350. [CrossRef]

8. Liu, T.; Fan, B.; Lu, J. Sediment-flow interactions at channel confluences: A flume study. Adv. Mech. Eng. 2015, 7, 1-9. [CrossRef]

9. Zhang, T.; $\mathrm{Xu}, \mathrm{W} . ; \mathrm{Wu}, \mathrm{C}$. Effect of discharge ratio on flow characteristics in $90^{\circ}$ equal-width open-channel junction. J. Hydrodyn. Ser. B 2009, 21, 541-549. [CrossRef]

10. Zeng, C.; Li, C.W. A hybrid RANS-LES model for combining flows in open-channel T-Junctions. J. Hydrodyn. Ser. B 2010, 22, 154-159. [CrossRef]

11. Ting, A.; Xu, W.L.; Wu, C. Hydrodynamics of right-angled channel confluences by a 2D numerical model. Iran. J. Sci. Tech. Trans. Civil Eng. 2011, 37, 271-283.

12. Riley, J.D.; Rhoads, B.L. Flow structure and channel morphology at a natural confluent meander bend. Geomorphology 2012, 163, 84-98. [CrossRef]

13. LIU, T.H.; Li, C.; FAN, B.L. Experimental study on flow pattern and sediment transportation at a $90^{\circ}$ open-channel confluence, Int. J. Sed. Res. 2012, 27, 178-187. [CrossRef]

14. Biswal, S.K.; Mohapatra, P.; Muralidhar, K. Hydraulics of combining flow in a right-angled compound open channel junction. Sadhana 2016, 41, 97-110. [CrossRef]

15. Sharifipour, M.; Bonakdari, H.; Zaji, A.H.; Shamshirband, S. Numerical investigation of flow field and flowmeter accuracy in open-channel junctions. Eng. App. Comput. Fluid Mech. 2015, 9, 280-290. [CrossRef]

16. Schindfessel, L.; Creëlle, S.; De Mulder, T. Flow patterns in an open channel confluence with increasingly dominant tributary inflow. Water 2015, 7, 4724-4751. [CrossRef]

17. Wang, X.; Wang, H.; Yan, X.; Liu, X.; Duan, H.F. Experimental Study on the Hydrodynamic Influence of River Flow Confluences to the Open Channel Stage-Discharge Relationship. Hydrologic. Sci. J. 2016, 64, 2025-2039. [CrossRef]

18. Sui, B.; Huang, S.H. Numerical analysis of flow separation zone in a confluent meander bend channel. J. Hydrodyn. Ser. B. 2017, 29, 716-723. [CrossRef]

19. Ramos, P.X.; Schindfessel, L.; Pêgo, J.P.; De Mulder, T. Influence of bed elevation discordance on flow patterns and head losses in an open-channel confluence, Water Sci. Eng. 2019, 12, 235-243.

20. Ramos, P.X.; Schindfessel, L.; Pêgo, J.P.; De Mulder, T. Flat vs. curved rigid-lid LES computations of an open-channel confluence. J. Hydroinform. 2019, 21, 318-334. [CrossRef]

21. Harlow, F.H.; Nakayama, P.I. Turbulence Transport Equations. Physic. Fluid. 1967, 10, 2323-2332. [CrossRef]

22. Launder, B.E.; Spaulding, D. Mathematical Models of Turbulence; Academic Press: London, UK; New York, NY, USA, 1972.

23. Hsu, C.C.; Lee, W.J.; Chang, C.H. Subcritical open-channel junction flow. J. Hydraul. Eng. 1998, 124, 847-855. [CrossRef]

(C) 2020 by the authors. Licensee MDPI, Basel, Switzerland. This article is an open access article distributed under the terms and conditions of the Creative Commons Attribution (CC BY) license (http://creativecommons.org/licenses/by/4.0/). 